\title{
Zero-range model of traffic flow
}

\author{
J. Kaupužs* \\ Institute of Mathematics and Computer Science, University of Latvia, LV-1459 Riga, Latvia \\ R. Mahnke ${ }^{\dagger}$ \\ Institut für Physik, Universität Rostock, D-18051 Rostock, Germany \\ R. J. Harris ${ }^{\ddagger}$ \\ Institut für Festkörperforschung, Forschungszentrum Jülich, D-52425 Jülich, Germany
}

(Received 26 April 2005; revised manuscript received 9 August 2005; published 21 November 2005)

\begin{abstract}
A multicluster model of traffic flow is studied, in which the motion of cars is described by a stochastic master equation. Assuming that the escape rate from a cluster depends only on the cluster size, the dynamics of the model is directly mapped to the mathematically well-studied zero-range process. Knowledge of the asymptotic behavior of the transition rates for large clusters allows us to apply an established criterion for phase separation in one-dimensional driven systems. The distribution over cluster sizes in our zero-range model is given by a one-step master equation in one dimension. It provides an approximate mean-field dynamics, which, however, leads to the exact stationary state. Based on this equation, we have calculated the critical density at which phase separation takes place. We have shown that within a certain range of densities above the critical value a metastable homogeneous state exists before coarsening sets in. Within this approach we have estimated the critical cluster size and the mean nucleation time for a condensate in a large system. The metastablity in the zero-range process is reflected in a metastable branch of the fundamental flux-density diagram of traffic flow. Our work thus provides a possible analytical description of traffic jam formation as well as important insight into condensation in the zero-range process.
\end{abstract}

DOI: 10.1103/PhysRevE.72.056125

PACS number(s): 89.40.-a, 02.50.Ey, 64.75.+g

\section{INTRODUCTION}

The development of traffic jams in vehicular flow is an everyday example of the occurrence of phase separation in low-dimensional driven systems, a topic which has attracted much recent interest (see, e.g., Refs. [1,2], and references therein). In Ref. [3] the existence of phase separation is related to the size dependence of domain currents and a quantitative criterion is obtained by considering the zero-range process (ZRP) as a generic model for domain dynamics. Phase separation corresponds to the phenomenon of condensation in the ZRP (see Ref. [4] for a recent review) whereby a macroscopic proportion of particles accumulate on a single site.

In this paper we use such a zero-range picture to study the phase separation in traffic flow. A stochastic master equation approach in the spirit of the probabilistic description of transportation [5] enables us to calculate the critical parameters of our model. We pay particular attention to the initial nucleation of a condensate in the ZRP, complementing previous work $[6,7]$ on the late-stage cluster coarsening. Significantly, we find that prior to condensation the system can exist in a homogeneous metastable state and we provide estimates of the critical cluster size and mean nucleation time. Finally, we apply these results to the description of traffic, obtaining a

\footnotetext{
*Electronic address: kaupuzs@latnet.lv

†Electronic address: reinhard.mahnke@uni-rostock.de

"Electronic address: r.harris@fz-juelich.de
}

fundamental flux-density diagram which includes a metastable branch. Metastability and hysteresis effects have been observed in real traffic, see, e.g., Refs. [8,9] for discussion of empirical data and the various different modeling approaches. For previous work focusing on the description of jam formation as a nucleation process, see Refs. [10,11].

\section{MODEL}

We consider a model of traffic flow, where cars are moving along a circular road. Each car occupies a certain length of road $\ell$. We divide the whole road of total length $L$ into cells of size $\ell$. Each cell can be either empty or occupied by a car, just as in cellular automation traffic models (see, e.g., Refs. [8,9], and references therein). Most such models use a discrete-time update rule, for example, see Ref. [12] for a class of traffic models related to a parallel updating version of the ZRP. In contrast, we consider the development of our system in continuous time. The probability per unit time for each car to move to the next cell is given by a certain transition rate, which depends on the actual configuration of empty and occupied cells. This configuration is characterized by the cluster distribution. An uninterrupted string of $n$ occupied cells, bounded by two empty cells, is called a cluster of size $n$. The clusters of size $n=1$ are associated with freely moving cars. The first car in each cluster is allowed to move forward by one cell. The transition rate $w_{n}$ of this stochastic event depends on the size $n$ of the cluster to which the car belongs. In this case $w_{1}$ is the mean of the inverse time necessary for a free car to move forward by one cell. The 
transition rate $w_{1}$ is related to the distribution of velocities in the free flow regime or phase, which is characterized by a certain car density $c_{\text {free }}$. For small densities, expected in the free flow phase in real traffic, the interaction between cars is weak and therefore the transition rate $w_{1}$ depends only weakly on the density $c_{\text {free }}$. Hence in the first approximation we may assume that $w_{1}$ is a constant.

This model can be directly mapped to the zero-range process (ZRP). Each vacancy (empty cell) in the original model is related to a box in the zero-range model. The number of boxes is fixed, and each box can contain an arbitrary number of particles (cars), which is equal to the size of the cluster located to the left (if cars are moving to the right) of the corresponding vacancy in the original model. If this vacancy has another vacancy to the left, then it means that the box is empty. Since the boundary conditions are periodic in the original model, they remain periodic also in the zero-range model. In this representation, one particle moves from a given box to the right with transition rate $w_{n}$, which depends only on the number of particles $n$ in this box. In the grand canonical ensemble, where the total number of particles is allowed to fluctuate, the stationary distribution over the cluster-size configurations is the product of independent distributions for individual boxes. The probability that there are just $n$ particles in a box in a homogeneous phase is $[13,14]$ $P(n) \propto z^{n} / \prod_{m=1}^{n} w_{m}$ for $n>0, P(0)$ being given by the normalization condition. Here $z=e^{\mu / k_{B} T}$ is the fugacity-a parameter which controls the mean number of particles in the system.

\section{MASTER EQUATION}

This result can be obtained and interpreted within the stochastic master equation approach [4]. Assuming the statistical independence of the distributions in different boxes, we have a multiplicative ansatz

$$
P_{2}(k, m, t)=P(k, t) P(m, t)
$$

for the joint probability $P_{2}(k, m, t)$ that there are $m$ particles in one box and $k$ particles in the neighboring box on the left at time $t$. This approximation leads to the mean-field dynamics described by the master equations [4]

$$
\begin{gathered}
\frac{\partial P(n, t)}{\partial t}=\langle w\rangle P(n-1, t)+w_{n+1} P(n+1, t) \\
-\left[\langle w\rangle+w_{n}\right] P(n, t), \quad n \geqslant 1, \\
\frac{\partial P(0, t)}{\partial t}=w_{1} P(1, t)-\langle w\rangle P(0, t),
\end{gathered}
$$

where

$$
\langle w\rangle(t)=\sum_{k=1}^{\infty} w_{k} P(k, t)
$$

is the mean inflow rate in a box. The ansatz (1) is an exact property of the stationary state of the grand canonical ensemble or, alternatively, of an infinitely large system [13]. Hence, in these cases, the master equations (2) and (3) give the exact stationary state while providing a mean-field approximation to the dynamics of reaching it.

The stationary solution $P(n)$ corresponding to $\partial P(n, t) / \partial t=0$ can be found recursively, starting from $n=0$. It yields the known result $[4,13,14]$

$$
P(n)=P(0)\langle w\rangle^{n} \prod_{m=1}^{n} \frac{1}{w_{m}}
$$

for $n>0$, where $P(0)$ is found from the normalization condition.

Denoting by $M$ the number of boxes, which corresponds to the number of vacancies in the original model, the mean number of cars on the road is given by $\langle N\rangle=M\langle n\rangle$, where

$$
\langle n\rangle=\sum_{n=1}^{\infty} n P(n)
$$

is the average number of particles in a box. Note that in the grand canonical ensemble the total number of cars as well as the length of the road $L$ fluctuate. For the mean value, measured in units of $\ell$, we have $\langle L\rangle=M+\langle N\rangle$. Hence the average density of cars is

$$
c=\frac{\langle N\rangle}{\langle L\rangle}=\frac{\langle n\rangle}{1+\langle n\rangle} .
$$

According to Eqs. (5), (6), and (7), we have the following relation:

$$
\frac{c}{1-c}=\frac{\sum_{n=1}^{\infty} n\langle w\rangle^{n} \prod_{m=1}^{n} \frac{1}{w_{m}}}{1+\sum_{n=1}^{\infty}\langle w\rangle^{n} \prod_{m=1}^{n} \frac{1}{w_{m}}}
$$

from which the stationary mean inflow rate $\langle w\rangle$ can be calculated at a given average density $c$.

\section{TRANSITION RATES AND PHASE SEPARATION}

Now we make the following choice for the transition rate dependence on the cluster size $n$ :

$$
w_{n}=w_{\infty}\left(1+\frac{b}{n^{\sigma}}\right) \text { for } n \geqslant 2,
$$

the value of $w_{1}$ being given separately, since it is related to the motion of uncongested cars, whereas $w_{n}$ with $n \geqslant 2$ represents the escaping from a jam of size $n$. Although an individual driver does not know how many cars are jammed behind him, the effective current of cars from a jam, represented by $w_{n}$, is a collective effect which is expected to depend on the correlations and internal struture (e.g., distribution of headways) within the cluster [3]. A monotonously decreasing dependence on cluster size, such as Eq. (9), can be considered as a type of slow-to-start rule-the longer a car has been stationary the larger the probability of a delay when starting (cf. Refs. [15-18]).

We now explore the consequences of the choice (9) in terms of the ZRP phase behavior and its implications for the 


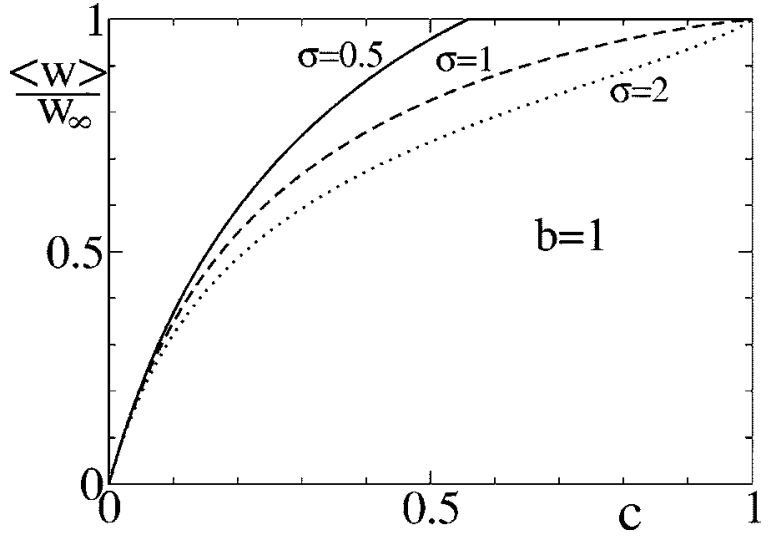

FIG. 1. $\langle w\rangle / w_{\infty}$ vs density $c$ at $b=1$ for different $\sigma$.

description of traffic flow. In numerical calculations we have assumed $w_{\infty}=1 / \tau_{\infty}=1$ and $w_{1}=5$, by choosing the time constant $\tau_{\infty}$ as a time unit, whereas the control parameters $b$ and $\sigma$ have been varied.

If $\sigma>1$, as well as for $b \leqslant 2$ at $\sigma=1$, Eq. (8) has a solution for any density $0<c<1$ (see dashed and dotted curves in Fig. 1).

This implies that the homogeneous phase is stable in the whole range of densities, i.e., there is no phase transition in a strict sense. If $\sigma<1$ (solid curve in Fig. 1), as well as for $b>2$ at $\sigma=1,\langle w\rangle / w_{\infty}$ reaches 1 at a critical density $0<c_{c r}$ $<1$, and there is no physical solution of Eq. (8) for $c>c_{c r}$. This means that the homogeneous phase cannot accommodate a larger density of particles and condensation takes place at $c>c_{c r}$.

This behavior underlies the known criterion for phase separation in one-dimensional driven systems [3]. For illustration, we comment that in the multicluster model considered in Ref. [19] the transition rates do not depend on the cluster sizes, only the inflow rate in a cluster depends on the overall car density and fraction of congested cars. This corresponds to the case $b=0$, where, according to the criterion, no macroscopic phase separation takes place-in agreement with the theoretical conclusions and simulation results of Ref. [19]. In contrast, a class of microscopic models was introduced in Ref. [20] where correlations within the domain (jam) give rise to currents of the form (9) with $\sigma=1$ and $b$ $>2$; phase separation is then observed.

At $c<c_{c r}$ in our model the cluster distribution function $P(n)$ decays exponentially fast for large $n$ whereas the decay is slower at $c=c_{c r}$. It is well known that the decay in this case is powerlike for $\sigma=1$, i.e., $P(n) \sim n^{-b}$ [3]. For $0<\sigma<1$, we find that the leading behavior is stretched exponential, i.e., $P(n) \sim \exp \left[-b n^{1-\sigma} /(1-\sigma)\right]$, in agreement with the result stated in Ref. [4]. Within our approach we have also derived the subleading terms which turn out to be relevant for 0 $<\sigma<1 / 2$. This calculation is presented in the Appendix and further illustrates the rich behavior of the model as $\sigma$ is varied.

At $\langle w\rangle / w_{\infty}=1$ the inflow $\langle w\rangle$ in a macroscopic cluster of size $n \rightarrow \infty$ is balanced with the outflow $w_{\infty}$. This means that at overall density $c>c_{c r}$ the homogeneous phase with density $c_{c r}$ is in equilibrium with a macroscopic cluster, repre-

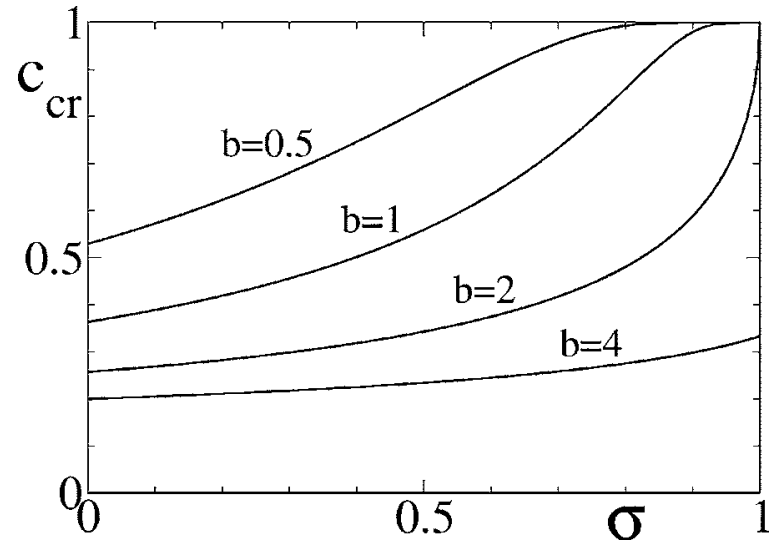

FIG. 2. Critical density as a function of control parameter $\sigma$ for different values of $b$.

sented by one of the boxes containing a nonvanishing fraction of all particles in the thermodynamic limit $[6,21]$. Hence $\langle w\rangle / w_{\infty}=1$ holds in the phase coexistence regime at $c>c_{c r}$.

According to Eq. (7), the critical density $c_{c r}$ is given by

$$
c_{c r}=\frac{\langle n\rangle_{c r}}{1+\langle n\rangle_{c r}},
$$

where $\langle n\rangle_{c r}$ is the mean cluster size at the critical density. Since $\langle w\rangle=w_{\infty}$ holds in this case, we have

$$
\langle n\rangle_{c r}=\frac{\sum_{n=1}^{\infty} n w_{\infty}^{n} \prod_{m=1}^{n} \frac{1}{w_{m}}}{1+\sum_{n=1}^{\infty} w_{\infty}^{n} \prod_{m=1}^{n} \frac{1}{w_{m}}} .
$$

The critical density, calculated numerically from Eqs. (10) and (11) as a function of parameters $\sigma$ and $b$, is shown in Figs. 2 and 3, respectively. In contrast to the situations discussed previously in the literature, in our model $w_{1}$ is not given by the general formula (9) but is an independent parameter. This distinction leads to quantitatively different re-

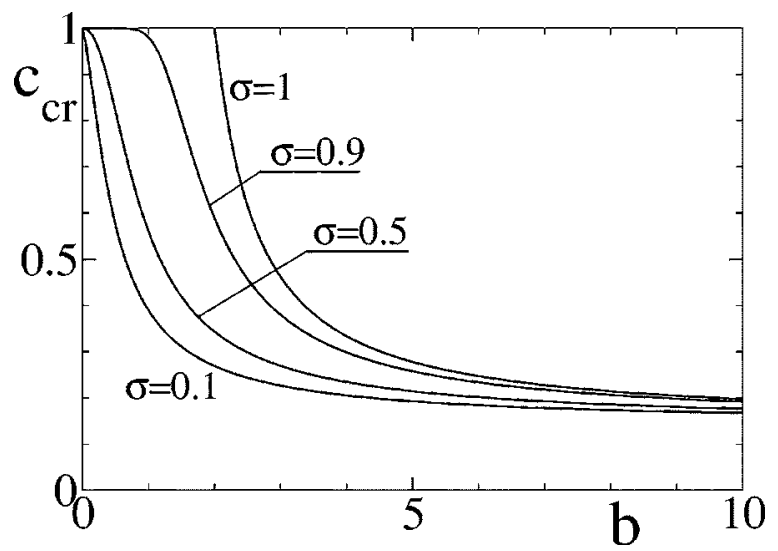

FIG. 3. Critical density as a function of control parameter $b$ for different values of $\sigma$. 
sults, for example, the critical density for $\sigma=1$ is analytically shown to be [22]

$$
c_{c r}=\frac{b(b+1)}{(b-1)\left[2(b+1)+w_{1}(b-2)\right]} .
$$

\section{METASTABILITY}

Suppose that at the initial time moment $t=0$ the system is in a homogeneous state with overall density slightly larger than $c_{c r}$. Here we study the development of such a state in the mean-field approximation provided by Eqs. (2) and (3). With this initial condition, the mean inflow rate in a box $\langle w\rangle$ is slightly larger than that at $c=c_{c r}$, i.e., $\langle w\rangle=w_{\infty}+\varepsilon$ holds with small and positive $\varepsilon$. Hence only large clusters with $w_{n}<w_{\infty}+\varepsilon$ have a stable tendency to grow, whereas any smaller cluster typically (except a rare case) fluctuates until it finally dissolves. In other words, the initially homogeneous system with no large clusters can stay in this metastable supersaturated state for a long time until a large stable cluster appears due to a rare fluctuation.

Neglecting the fluctuations, the time development of the size $n$ of a cluster is described by the deterministic equation

$$
\frac{d n}{d t}=\langle w\rangle-w_{n} .
$$

According to this equation, the undercritical clusters with $n$ $<n_{c r}$ tend to dissolve, whereas the overcritical ones with $n$ $>n_{c r}$ tend to grow, where the critical cluster size $n_{c r}$ is given by the condition

$$
\langle w\rangle=w_{n_{c r}} .
$$

Using Eq. (9) yields

$$
n_{c r} \simeq\left(\frac{b}{\langle w\rangle / w_{\infty}-1}\right)^{1 / \sigma} .
$$

In this case $n_{c r}$ is rounded to an integer value.

This deterministic approach describes only the most probable scenario for an arbitrarily chosen cluster of a given size. It does not allow one to obtain the distribution over cluster sizes: the deterministic equation (13) suggests that all clusters shrink to zero size if they are smaller than $n_{c r}$ at the beginning, whereas the real size distribution arises from the competition between opposite stochastic events of shrinking and growing. Assuming that the distribution of relatively small clusters contributing to $\langle n\rangle$ is quasistationary, i.e., that the detailed balance [equality of the terms in Eqs. (2) and (3) describing opposite stochastic events] for these clusters is almost reached before any cluster with $n>n_{c r}$ has appeared, we have

$$
\langle n\rangle \simeq \sum_{n=1}^{n_{c r}} n P(n)
$$

for such a metastable state. In this case from Eq. (7) we obtain

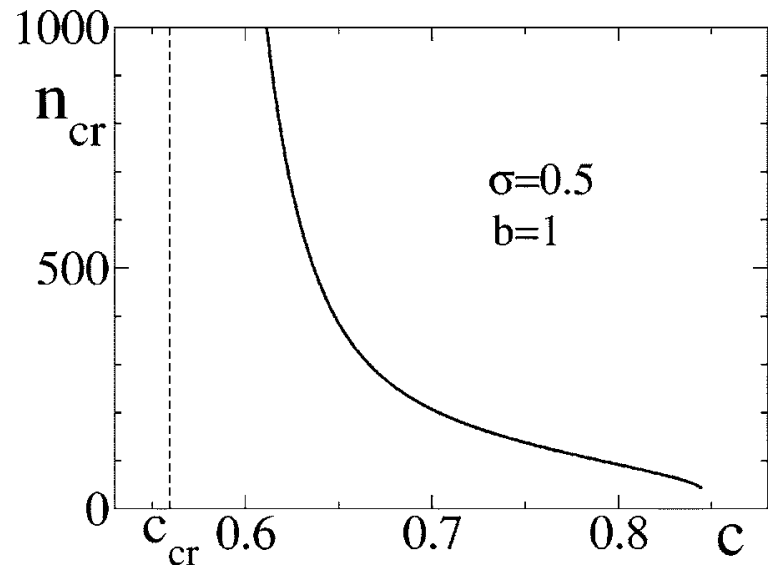

FIG. 4. Critical cluster size vs density at $\sigma=0.5$ and $b=1$. The critical density is indicated by a vertical dashed line.

$$
\frac{c}{1-c} \simeq \frac{\sum_{n=1}^{n_{c r}} n\langle w\rangle^{n} \prod_{m=1}^{n} \frac{1}{w_{m}}}{1+\sum_{n=1}^{n_{c r}}\langle w\rangle^{n} \prod_{m=1}^{n} \frac{1}{w_{m}}}
$$

instead of Eq. (8) for calculation of $\langle w\rangle$ in this homogeneous metastable state. The critical cluster size is found self consistently solving Eqs. (15) and (17) as a system of equations. From Eq. (15) we see that the critical cluster size $n_{c r}$ diverges at $c \rightarrow c_{c r}$, since $\langle w\rangle \rightarrow w_{\infty}$. The results of calculation of $n_{c r}$ (with rounding down to an integer value) at $\sigma=0.5, b=1$ and at $\sigma=1, b=3$ are shown in Figs. 4 and 5, respectively.

\section{MEAN NUCLEATION TIME}

The nucleation time, i.e., the mean time the system stays in the homogeneous metastable state, is considered for the zero-range process. A characteristic time scale for a particle to escape from a jam has been evaluated in Ref. [15] for the related bus route model. This time scale, however, refers to already formed finite-size jams and is therefore different to the nucleation time we are interested in.

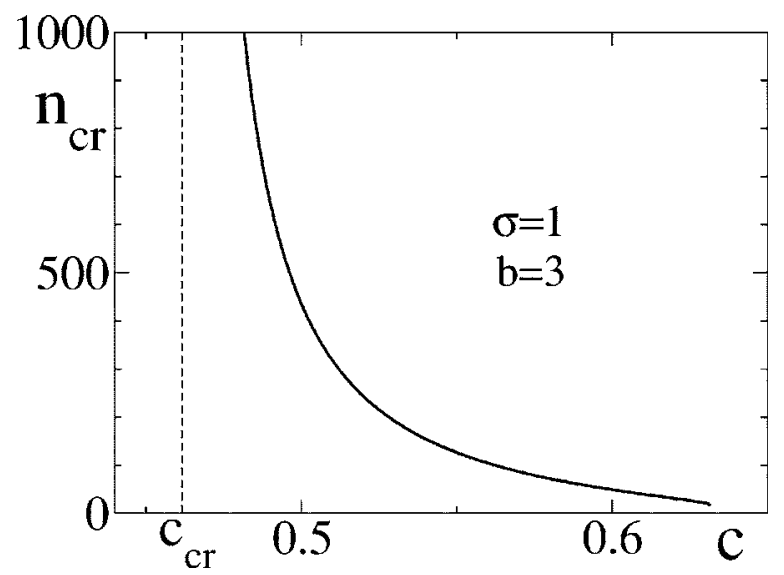

FIG. 5. Critical cluster size vs density at $\sigma=1$ and $b=3$. The critical density is indicated by a vertical dashed line. 
Within the framework of mean-field dynamics, the mean nucleation time in our model can be evaluated as follows. Let $\mathcal{P}(t)$ be the probability density of the first passage time to exceed the critical number of particles $n_{c r}$ in a single box. By our definition, the nucleation occurs when one of the $M$ boxes reaches the cluster size $n_{c r}+1$. The probability that it occurs first in a given box within a small time interval $[t ; t$ $+d t]$ is thus $\mathcal{P}(t) d t \times\left[1-\int_{0}^{t} \mathcal{P}\left(t^{\prime}\right) d t^{\prime}\right]^{M-1}$ according to our assumption that the boxes are statistically independent. The term $\left[1-\int_{0}^{t} \mathcal{P}\left(t^{\prime}\right) d t^{\prime}\right]^{M-1}$ is the probability that in all other boxes, except the given one, the overcritical cluster size $n_{c r}$ +1 has still not been reached. Since the nucleation can occur in any of $M$ boxes, the nucleation probability density $\mathcal{P}_{M}(t)$ for the system of $M$ boxes is given by

$$
\begin{aligned}
\mathcal{P}_{M}(t) & =M \mathcal{P}(t) \times\left[1-\int_{0}^{t} \mathcal{P}\left(t^{\prime}\right) d t^{\prime}\right]^{M-1} \\
& \simeq M \mathcal{P}(t) \exp \left(-M \int_{0}^{t} \mathcal{P}\left(t^{\prime}\right) d t^{\prime}\right)
\end{aligned}
$$

The latter equality holds for large $M$, since all $M$ boxes are equivalent, and therefore the probability $\int_{0}^{t} \mathcal{P}\left(t^{\prime}\right) d t^{\prime}$ that the nucleation occurs in a given box within a characteristic time interval $t \sim\langle T\rangle_{M}$ is a small quantity of order $1 / M$. The mean nucleation time for the system of $M$ boxes is

$$
\langle T\rangle_{M}=\int_{0}^{\infty} t \mathcal{P}_{M}(t) d t
$$

Here $\langle T\rangle_{1}$ is the mean first passage time for a single box.

In order to estimate $\langle T\rangle_{M}$ according to Eqs. (18) and (19), one needs some idea about the first passage time probability density for one box $\mathcal{P}(t)$. This is actually the problem of a particle escaping from a potential well. Since we start with an almost homogeneous state of the system, we may assume zero cluster size $n=0$ as the initial condition. The first passage time probability density can be calculated as the probability per unit time to reach the state $n_{c r}+1$, assuming that the particle is absorbed there. It is reasonable to assume that after a certain equilibration time $t_{e q}$, when a quasistationary distribution of the cluster sizes within $n \leqslant n_{c r}$ is reached, the escaping from this region is characterized by a certain transition rate $w_{e s c}$. Hence for $t>t_{e q}$ we have

$$
\mathcal{P}(t) \simeq w_{\text {esc }} \times\left[1-\int_{0}^{t} \mathcal{P}\left(t^{\prime}\right) d t^{\prime}\right],
$$

where the expression in square brackets is the probability that the absorption at $n_{c r}+1$ has still not occurred up to the time moment $t$. At high enough potential barriers (large mean first passage times) the short-time contribution to the integral is irrelevant and, by means of Eq. (19), the solution of Eq. (20) can be written as

$$
\mathcal{P}(t)=\frac{1}{\langle T\rangle_{1}} \exp \left(-\frac{t}{\langle T\rangle_{1}}\right),
$$

where $\langle T\rangle_{1}=w_{e s c}^{-1}$. Obviously, this approximate solution of the
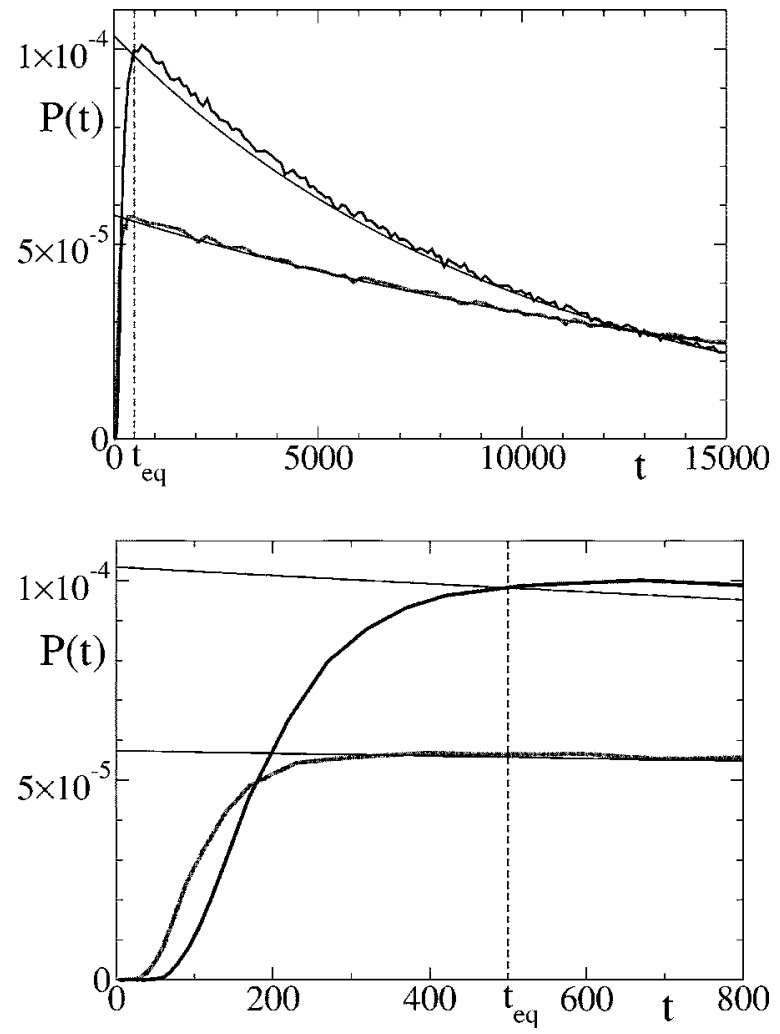

FIG. 6. Comparison between the theoretical approximation (21) for $\mathcal{P}(t)$ (smooth curves) and mean-field simulation results (fluctuating curves) shown in a longer (top) and in a shorter (bottom) time scale. The vertical dashed line indicates the equilibration time $t_{e q}$ $\approx 500$ for the set of parameters $\sigma=0.5, b=1$, and $c=0.84$ represented by the upper curves in both pictures. The other curves correspond to $\sigma=1, b=3$, and $c=0.61$.

first passage problem is not valid for very short times $t$ $\ll t_{e q}$, since the short-time solution should explicitly depend on the initial condition. In particular, if we start at $n=0$, then the state $n_{c r}+1$ cannot be reached immediately, so that $\mathcal{P}(0)=0$. Nevertheless Eq. (21) can be used to estimate the mean nucleation time $\langle T\rangle_{M}$ provided that $\langle T\rangle_{M}>t_{e q}$.

We have checked the correctness of these theoretical expectations within the mean-field dynamics represented by Eqs. (2) and (3) by comparing them with the results of simulation of stochastic trajectories generated according to these equations. The simulation curves for $\mathcal{P}(t)$ at two different sets of parameters: $\sigma=0.5, b=1, c=0.84$ (with $n_{c r}=48$ ), and $\sigma=1, b=3, c=0.61$ (with $n_{c r}=35$ ) are shown in Fig. 6 in two different time scales. As we see, Eq. (21) is a good approximation for large enough times $t>t_{e q}$. For definiteness, we have identified the equilibration time $t_{e q}$ with the crossing point of the theoretical and simulated curves. An interesting additional feature is the presence of an apparent nucleation time lag, which is about $t_{\text {lag }} \approx 60$ for the first set of parameters and about $t_{\text {lag }} \approx 30$ for the second one. Evidently, the first passage time probability density $\mathcal{P}(t)$ tends to zero very rapidly when $t$ decreases below $t_{\text {lag }}$.

By inserting Eq. (21) in Eq. (19) we obtain 


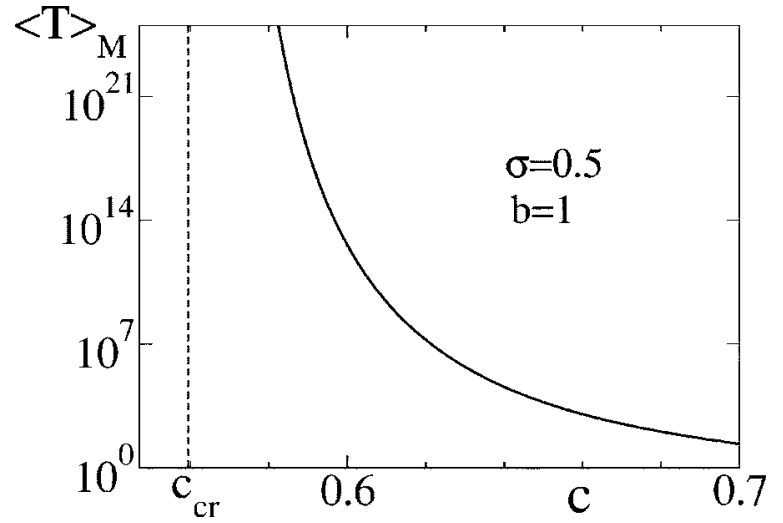

FIG. 7. Mean nucleation time vs density at $\sigma=0.5, b=1$, and $M=10^{6}$. The critical density is indicated by a vertical dashed line.

$$
\langle T\rangle_{M} \simeq M\langle T\rangle_{1} \int_{0}^{\infty} x e^{-x} \exp \left(-M\left[1-e^{-x}\right]\right) d x
$$

after changing the integration variable $t /\langle T\rangle_{1} \rightarrow x$. Taking into account that only the region $x \sim 1 / M$ contributes to the integral at large $M$, we arrive at a very simple expression,

$$
\langle T\rangle_{M} \simeq \frac{\langle T\rangle_{1}}{M}
$$

relating the mean first passage time or nucleation time in a system of $M$ boxes with that of one box. The latter can be calculated easily by the known formula [23]

$$
\langle T\rangle_{1}=\sum_{n=0}^{n_{c r}}[\langle w\rangle \widetilde{P}(n)]^{-1} \sum_{m=0}^{n} \widetilde{P}(m),
$$

where $\widetilde{P}(0)=1$ and $\widetilde{P}(n)=\prod_{k=1}^{n}\left(\langle w\rangle / w_{k}\right)$ with $n>1$ represent the unnormalized stationary probability distribution.

The mean nucleation time versus density $c$, calculated from Eqs. (23) and (24) at $M=10^{6}$, is shown in Figs. 7 and 8. Figure 7 refers to the case $\sigma=0.5$ and $b=1$, whereas Fig. 8 is for the case $\sigma=1$ and $b=3$. These figures show that the mean

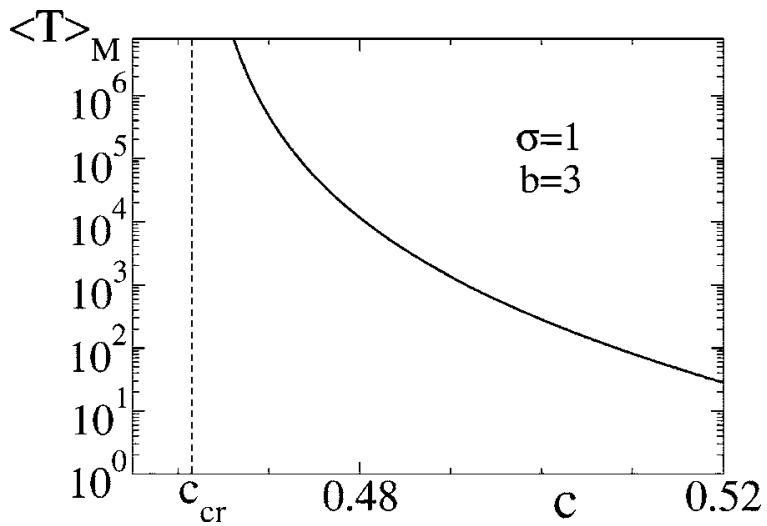

FIG. 8. Mean nucleation time vs density at $\sigma=1, b=3$, and $M$ $=10^{6}$. The critical density is indicated by a vertical dashed line.

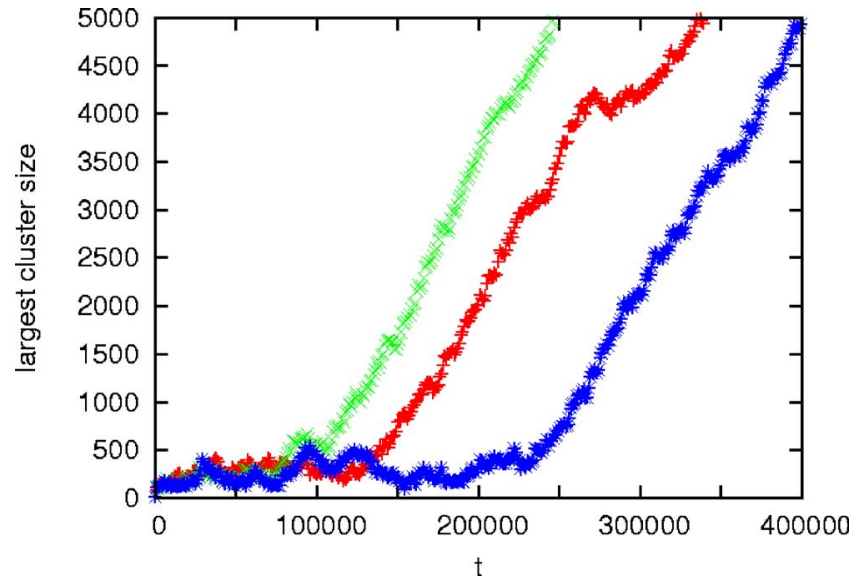

FIG. 9. (Color online) Largest cluster size vs time for $\sigma$ $=0.5, b=1, w_{1}=5, c=0.66, M=10^{5}$. Results from three independent Monte Carlo runs are shown.

nucleation time increases dramatically as the critical cluster size $n_{c r}$ increases (see the corresponding plots in Figs. 4 and 5) approaching the critical density $c_{c r}$.

According to our previous discussion, estimate (23) is valid for large enough mean nucleation times $\langle T\rangle_{M}>t_{e q}$, in particular, when approaching the critical density $c \searrow c_{c r}$ at any large but fixed $M$. It is not valid in the thermodynamic limit $M \rightarrow \infty$ at a fixed density $c$-Eq. (23) suggests that $\langle T\rangle_{M}$ decreases as $\sim 1 / M$, whereas in reality the decrease must be slower for small nucleation times (large $M$ ) since $\mathcal{P}(t) \rightarrow 0$ as $t \rightarrow 0$. In particular, the mean-field dynamics suggests that for a wide range of $M$ values $\langle T\rangle_{M}$ quasisaturates at $\langle T\rangle_{M} \approx t_{\text {lag }}$, since the critical cluster size is almost never reached before $t=t_{\text {lag }}$.

\section{SIMULATION RESULTS}

Numerical simulations of the zero-range model show clear evidence for the existence of a metastable state prior to condensation. In Fig. 9 we show the largest cluster size as a function of time for three separate Monte Carlo runs in the case $\sigma=0.5, b=1, w_{1}=5, M=10^{5}$.

For each run the system was started in a random uniform initial condition with density $c=0.66$ (for these parameters $c_{c r} \simeq 0.56$ ). It is clearly seen that after a short equilibriation period the system fluctuates in a metastable state before a condensate appears. The critical cluster size is observed to be around 400 in good agreement with the prediction $n_{c r} \simeq 330$ from Eqs. (15) and (17) (see Fig. 4). However, the metastable time is about an order of magnitude larger than predicted.

In Fig. 10 we show the distribution of cluster sizes (for small clusters) averaged over the metastable state of one such run. The distribution is in good agreement with Eq. (5) with $\langle w\rangle=w_{n_{c r}}$, thus supporting the assumption of quasistationarity.

In the analytical treatment of the previous section we calculated the mean time for the maximum cluster size to exceed $n_{c r}$ under the assumption that the current in the meta- 


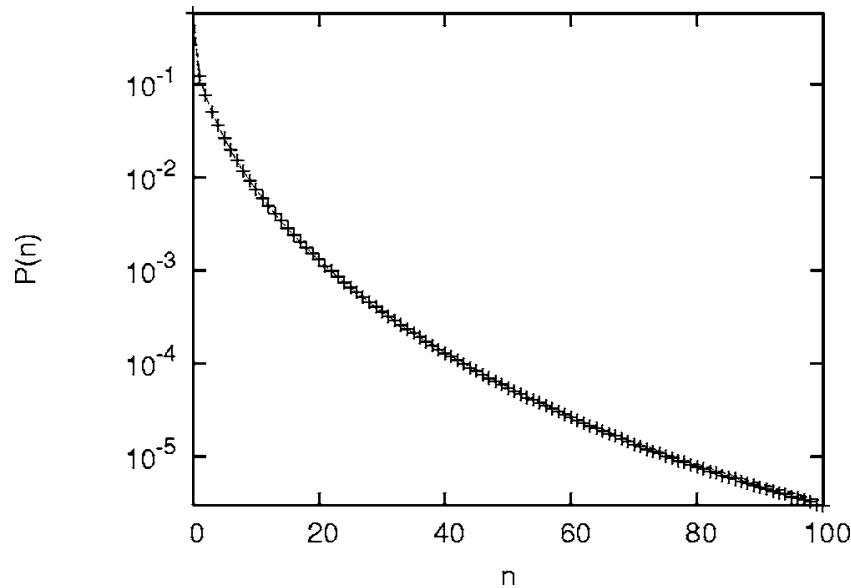

FIG. 10. Distribution over small cluster sizes in metastable state: results for Monte Carlo simulation (crosses) compared to prediction of Eq. (5) with $\langle w\rangle=w_{n_{c r}}$ and $n_{c r}$ calculated numerically from Eqs. (15) and (17) (dashed line).

stable state is constant. In practice, of course, the metastable current also fluctuates (and the fluctations are greater when $w_{n}$ depends more strongly on cluster size, e.g., for the $\sigma=1$ case compared to, say, $\sigma=0.5$ ). Simulations suggest that these fluctuations can destroy the metastable state in cases where the metastable current $w_{n_{c r}}$ is close to the current of the condensed phase $w_{\infty}$.

In contrast, for parameters where the metastable state is well separated from the condensed state we find relatively good quantitive agreement between theory and simulation. For example, in Figs. 11 and 12 we compare the average simulation values of critical cluster size and nucleation time to the theoretical predictions for a range of densities in the case $\sigma=0.5$ and $b=3$.

In these simulations we crudely identified the end of the metastable state as the point when the current out of the largest cluster had been less than the average system current for 50 consecutive Monte Carlo time steps.

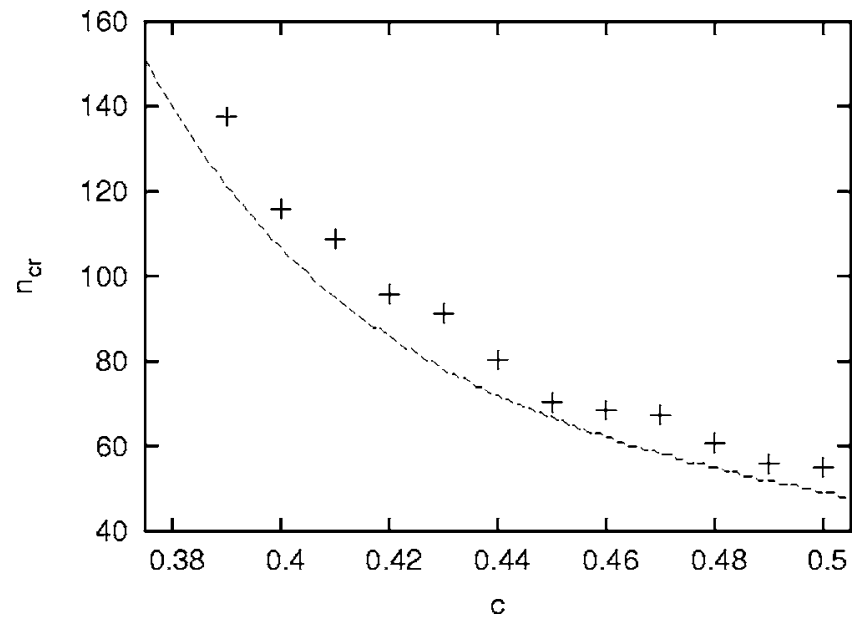

FIG. 11. Critical cluster size vs density for $\sigma=0.5, b=3, w_{1}$ $=5, M=10^{5}\left(c_{c r} \simeq 0.27\right)$. Crosses show simulation data (averaged over ten Monte Carlo histories), dashed line is prediction of Eqs. (15) and (17).

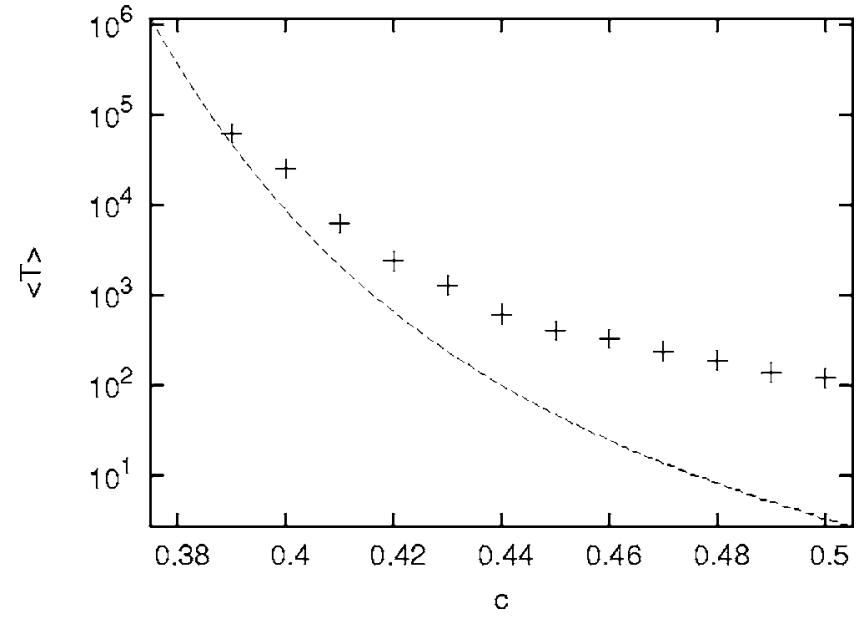

FIG. 12. Nucleation time vs density for $\sigma=0.5, b=3, w_{1}$ $=5, M=10^{5}\left(c_{c r} \simeq 0.27\right)$. Crosses show simulation data (averaged over ten Monte Carlo histories), the dashed line is the prediction of Eqs. (23) and (24).

We find that our mean-field theory fairly accurately reproduces the critical cluster size but systematically underestimates the nucleation time. This discrepancy may be partly due to the presence of (weak) dynamical correlations between the numbers of particles in the boxes in the fluctuating metastable state. Namely, the appearance of a large cluster with $n \simeq n_{c r}$ is likely to be accompanied by a slight depletion of the surrounding medium. Furthermore, we only calculated the mean first passage time and ignored the probability that a cluster reaches $n_{c r}+1$ and is immediately driven by a fluctuation back below $n_{c r}$. Monte Carlo histories which involve such a fluctuation back into the metastable state before a condensate is established would increase the average simulation nucleation time above the theoretical prediction.

Despite the neglect of current fluctuations, dynamical correlations, etc., our simulations show that the simple meanfield approach provides a good qualitative description of the metastable state and its dependence on density. It thus represents an important first step towards more refined theories.

\section{CONCLUSIONS}

In this paper we have studied a zero-range model motivated by the observation of phase separation in traffic flow. In particular, we have demonstrated the existence of a metastable state which exists prior to condensation and obtained analytical estimates for the critical cluster size and mean nucleation time. This insight into the mechanism of cluster formation in the ZRP is supported by the simulation results discussed in the previous section. In the context of traffic flow we now conclude by further exploring the significance of this metastable state.

The relation between density $c$ and flux $j$ of cars is known as the fundamental diagram of traffic flow. The average stationary flux can be calculated as follows:

$$
j=\sum_{n=1}^{\infty} Q(n) w_{n},
$$

where $Q(n)$ is the probability that there is a car in a given cell (in the original model) which can move forward with the 

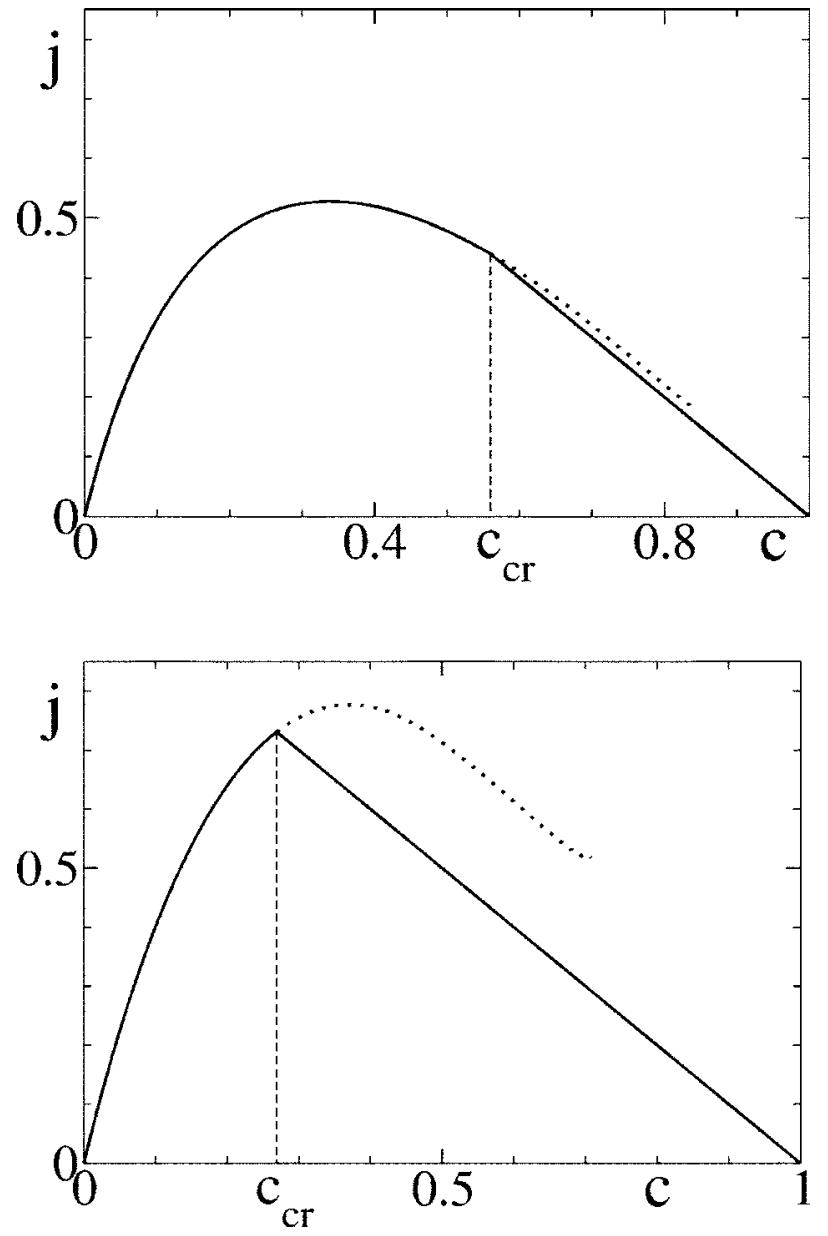

FIG. 13. The fundamental (flux-density) diagram for two different sets of control parameters: $\sigma=0.5, b=1, w_{1}=5$ (top); $\sigma$ $=0.5, b=3, w_{1}=5$ (bottom). The branches of metastable homogeneous state are shown by dotted lines, the critical densities $c_{c r}$ are indicated by vertical dashed lines.

rate $w_{n}$. Note that only those cars contribute to the flux, which are the first in some cluster. Hence $Q(n)$ $=\varphi P(n) / \Sigma_{m=1}^{\infty} P(m)$, where $\varphi$ is the fraction of cells, which contain such cars. This fraction can be calculated easily as the number of clusters divided by the total number of cells. These quantities fluctuate in our model. For large systems, however, they can be replaced by the mean values. The mean number of clusters is equal to the mean number of nonempty boxes $M \sum_{n=1}^{\infty} P(n)$ in the zero-range model, whereas the mean number of cells, i.e., the mean length of the road is $\langle L\rangle=M+\langle N\rangle=M(1+\langle n\rangle)=M /(1-c)$, as we have already discussed in Sec. III. Hence $Q(n)=(1-c) P(n)$ and Eq. (25) reduces to

$$
j=(1-c)\langle w\rangle .
$$

The mean stationary transition rate $\langle w\rangle$ depends on the car density $c$. For undercritical densities $c<c_{c r}$, this quantity is the solution of Eq. (8). For overcritical densities we have $\langle w\rangle=w_{\infty}$ in the phase coexistence regime, as discussed in Sec. IV, therefore in this case the fundamental diagram reduces to a straight line

$$
j=(1-c) w_{\infty}, \quad c \geqslant c_{c r} .
$$

In the metastable homogeneous state at $c>c_{c r}$ the mean transition rate $\langle w\rangle$ together with the critical cluster size $n_{c r}$ can be found from the system of equations (15) and (17), which allows calculation of the metastable branch of flux $j$.

The resulting fundamental diagrams for $\sigma=0.5, w_{1}=5$ and two values of parameter $b$ are shown in Fig. 13. As we see, the shape of the fundamental diagram, as well as the critical density and location of the metastable branch depend remarkably on the value of $b$. These features will also depend on the values of $\sigma$ and $w_{1}$. The metastable branch ends abruptly at certain density above which Eqs. (15) and (17) have no real solution. It corresponds to a relatively small, but finite value of the critical cluster size $n_{c r}$.

Note that a metastable branch is also observed in simulations of cellular automata with slow-to-start rules [16-18]. In our examples, however, the metastable branch is located at larger densities and decreases with increasing of $c$ over a certain wide range of values depending on $b, \sigma$, and $w_{1}$. The simulations of the previous section suggest that when this metastable branch is well separated from the condensed section of the fundamental diagram, our picture is robust even in the presence of fluctuations.

In summary therefore we believe that by suitable variation of parameters our simple model can reproduce some important features of real traffic flow. There is much scope for further investigation, both analytical and computational.

\section{ACKNOWLEDGMENTS}

One of us (J.K.) acknowledges support by the German Science Foundation (DFG) via Project No. 436 LET 17/3/05. The main part of the research was done at Rostock University, Institute of Physics. We are grateful to G. M. Schütz (Jülich) for helpful discussions and comments on the manuscript.

\section{APPENDIX: CRITICAL CLUSTER DISTRIBUTION FOR $0<\sigma<1$}

Here we focus on the cluster size distribution at $c=c_{c r}$ in the case $0<\sigma<1$, presenting a detailed calculation of the asymptotic behavior for large $n$.

According to Eq. (5), at $c=c_{c r}$ where $\langle w\rangle=w_{\infty}$, we have

$$
\begin{aligned}
\ln P(n)= & \ln \left(\frac{P(0) w_{\infty}}{w_{1}}\right)-\sum_{m=2}^{n} \ln \left(1+\frac{b}{m^{\sigma}}\right)=\ln \left(\frac{P(0) w_{\infty}}{w_{1}}\right) \\
& -\int_{1}^{n} \ln \left(1+\frac{b}{m^{\sigma}}\right) d m+C+\delta(n),
\end{aligned}
$$

where $C$ is a constant, and $\delta(n) \rightarrow 0$ at $n \rightarrow \infty$. The latter follows from the fact that each term with $m=k$ in the sum generates terms $\propto k^{-y}$ with $y>0$ when the logarithm is expanded in Taylor series, and for each of these terms we have $k^{-y}=\int_{k-1}^{k} m^{-y} d m+O\left(k^{-y-1}\right)$ at $k \rightarrow \infty$. It ensures that the difference between the integral and the sum in Eq. (A1) is finite and tends to some constant $C$ at $n \rightarrow \infty$ for $\sigma>0$. The remain- 
der term $\delta(n)$ is irrelevant for the leading asymptotic behavior of $P(n)$. By expanding the logarithm and integrating term by term, for $0<\sigma<1$ and $\sigma \neq \frac{1}{2}, \frac{1}{3}, \frac{1}{4}, \ldots$ we obtain

$$
P(n) \propto \prod_{k=1}^{[1 / \sigma]} \exp \left\{\frac{(-b)^{k}}{k} \frac{n^{1-k \sigma}}{1-k \sigma}\right\},
$$

where $[1 / \sigma]$ denotes the integer part of $1 / \sigma$. The cases where $\sigma$ is an inverse integer are special, since a term $\propto 1 / m$ appears in the expansion of the logarithm, giving rise to a powerlike correction to the stretched exponential behavior, viz.

$$
P(n) \propto n^{\sigma(-b)^{1 / \sigma}} \prod_{k=1}^{\sigma^{-1}-1} \exp \left\{\frac{(-b)^{k}}{k} \frac{n^{1-k \sigma}}{1-k \sigma}\right\}
$$

for $\sigma=\frac{1}{2}, \frac{1}{3}, \frac{1}{4}, \ldots$. The known result for $\sigma=1$ can also be obtained by this method: it corresponds to the power-like prefactor in Eq. (A3). Only the linear expansion term of the logarithm is relevant at $1 / 2<\sigma<1$, so we find $P(n)$ $\propto \exp \left[-b n^{1-\sigma} /(1-\sigma)\right]$ in the limit of large $n$. The first two terms are relevant for $1 / 3<\sigma \leqslant 1 / 2$, the third one becomes important for $1 / 4<\sigma \leqslant 1 / 3$, and so on. Equations (A2) and (A3) represent an exact analytical result at $n \rightarrow \infty$ which we have also verified numerically at different values of $\sigma$ and $b$. In this form, where the proportionality coefficient is not specified, Eqs. (A2) and (A3) are universal, i.e., they do not depend on the choice of $w_{1}$.
[1] D. Mukamel, in Soft and Fragile Matter: Nonequilibrium Dynamics, Metastability and Flow, edited by M. E. Cates and M. R. Evans (Institute of Physics Publishing, Bristol, 2000).

[2] G. M. Schütz, J. Phys. A 36, R339 (2003).

[3] Y. Kafri, E. Levine, D. Mukamel, G. M. Schütz, and J. Török, Phys. Rev. Lett. 89, 035702 (2002).

[4] M. R. Evans and T. Hanney, J. Phys. A 38, R195 (2005).

[5] R. Mahnke, J. Kaupužs, and I. Lubashevsky, Phys. Rep. 408, 1 (2005).

[6] S. Grosskinsky, G. M. Schütz, and H. Spohn, J. Stat. Phys. 113, 389 (2003).

[7] C. Godrèche, J. Phys. A 36, 6313 (2003).

[8] D. Chowdhury, L. Santen, and A. Schadschneider, Phys. Rep. 329, 199 (2000).

[9] D. Helbing, Rev. Mod. Phys. 73, 1067 (2001).

[10] R. Mahnke and J. Kaupužs, Phys. Rev. E 59, 117 (1999).

[11] R. Mahnke, J. Kaupužs, and V. Frishfelds, Atmos. Res. 65, 261 (2003).

[12] E. Levine, G. Ziv, L. Gray, and D. Mukamel, Physica A 340,
636 (2004).

[13] F. Spitzer, Adv. Math. 5, 246 (1970).

[14] M. R. Evans, Braz. J. Phys. 30, 42 (2000).

[15] O. J. O'Loan, M. R. Evans, and M. E. Cates, Phys. Rev. E 58, 1404 (1998).

[16] M. Takayasu and H. Takayasu, Fractals 1, 860 (1993).

[17] S. C. Benjamin, N. F. Johnson, and P. M. Hui, J. Phys. A 29, 3119 (1996).

[18] R. Barlovic, L. Santen, A. Schadschneider, and M. Schreckenberg, Eur. Phys. J. B 5, 793 (1998).

[19] J. Kaupužs and R. Mahnke, Eur. Phys. J. B 14, 793 (2000).

[20] Y. Kafri, E. Levine, D. Mukamel, G. M. Schütz, and R. D. Willmann, Phys. Rev. E 68, 035101(R) (2003).

[21] I. Jeon, P. March, Can. Math. Soc. Conf. Proc. 26, 233 (2000).

[22] G. M. Schütz (private communication).

[23] C. W. Gardiner, Handbook of Stochastic Methods for Physics, Chemistry, and the Natural Sciences (Springer, Berlin, 1983, 1994). 